\title{
Brown adipose tissue identification in an adult human using IDEAL MRI
}

\author{
Narendra L Reddy 1 , Terence A Jones ${ }^{1}$, Sarah C Wayte ${ }^{2}$, Oludolapo Adesanya ${ }^{3}$, Yen Yeo ${ }^{4}$, Gyanendra \\ Tripathi', Philip G McTernan ${ }^{1}$, Harpal S Randeva1, Sudhesh Kumar'1, Charles E Hutchinson ${ }^{1,3}$, \\ Thomas M Barber ${ }^{1}$ \\ Warwick Medical School'1, University of Warwick, Clinical Sciences Research Laboratories, Coventry, UK \\ Departments of Medical Physics ${ }^{2}$, Radiology ${ }^{3}$ \& Histopathology ${ }^{4}$, University Hospitals of Coventry \& Warwickshire, UK
}

\begin{abstract}
Aim
Manipulation of human brown adipose tissue (BAT) represents a novel therapeutic option for diabesity. The aim of our study was to develop and test a novel Magnetic Resonance Imaging (MR) based method to identify human BAT and delineate it from white adipose tissue (WAT), and validate it by providing immuno-histochemical confirmation.
\end{abstract}

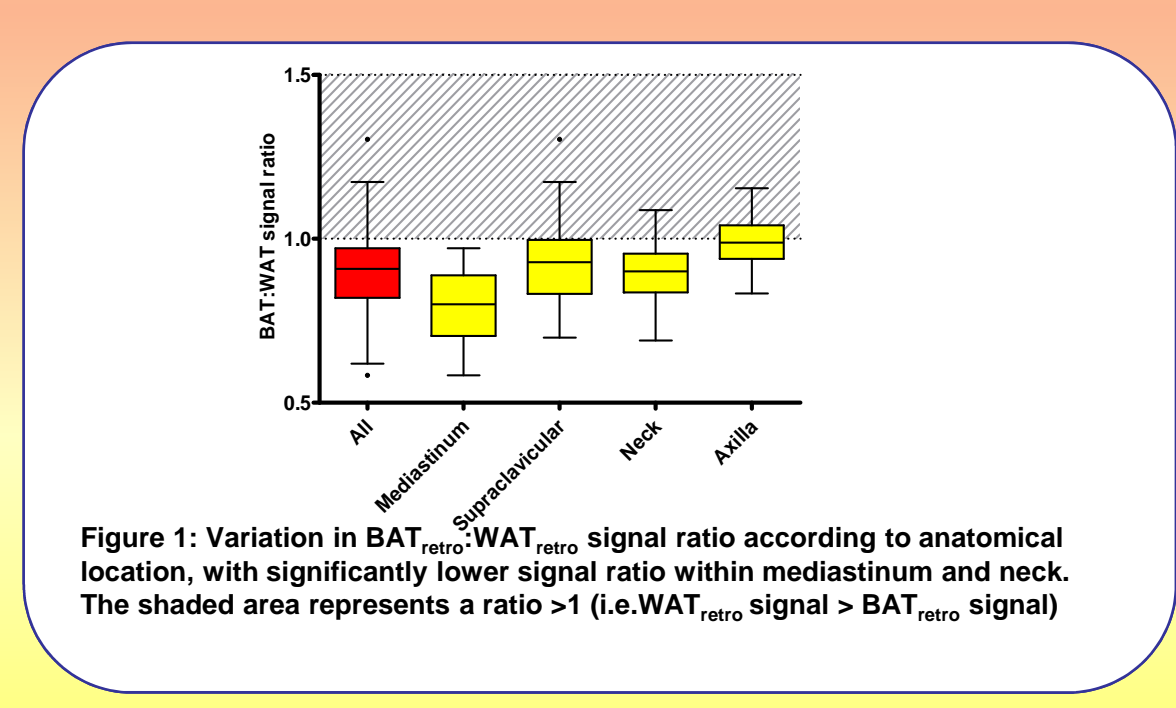

\section{Methodology}

Initial scanning with ${ }^{18}$ F-FDG PET-CT radiotracer uptake on a 25-year old Caucasian female with primary hyperparathyroidism, showed avid uptake within the mediastinum, neck, supraclavicular fossae and axillae, consistent with BAT. Subsequently, serial MR scans were performed using 3-echo IDEAL (iterative decomposition of water and fat with echo asymmetry and least-squares estimation) sequence. Retrospectively, regions of interest (ROIs) were identified on MR corresponding to PET-CT images. Prospectively, ROls were identified on MR images based on signal intensity and appearance, and compared with PET-CT. Immunohistochemical staining using uncoupling protein-1 antibody was performed on fat samples corresponding to low MR-signal, obtained during parathyroidectomy.

\section{Results}

Of the 111 retrospectively identified ROls from PET-CT scans, $88(79 \%)$ showed corresponding low signal on the MR images: $100 \%$ in mediastinum, $29 / 31$ (93.5\%) in neck, 31/41 (75.6\%) supraclavicular, and $8 / 14(57 \%)$ in axillae. Prospectively, $47 / 54$ (87\%) of ROls identified on MR scans corresponded to increased areas of uptake on PET-CT scans. Histology and immunohistochemistry confirmed BAT.

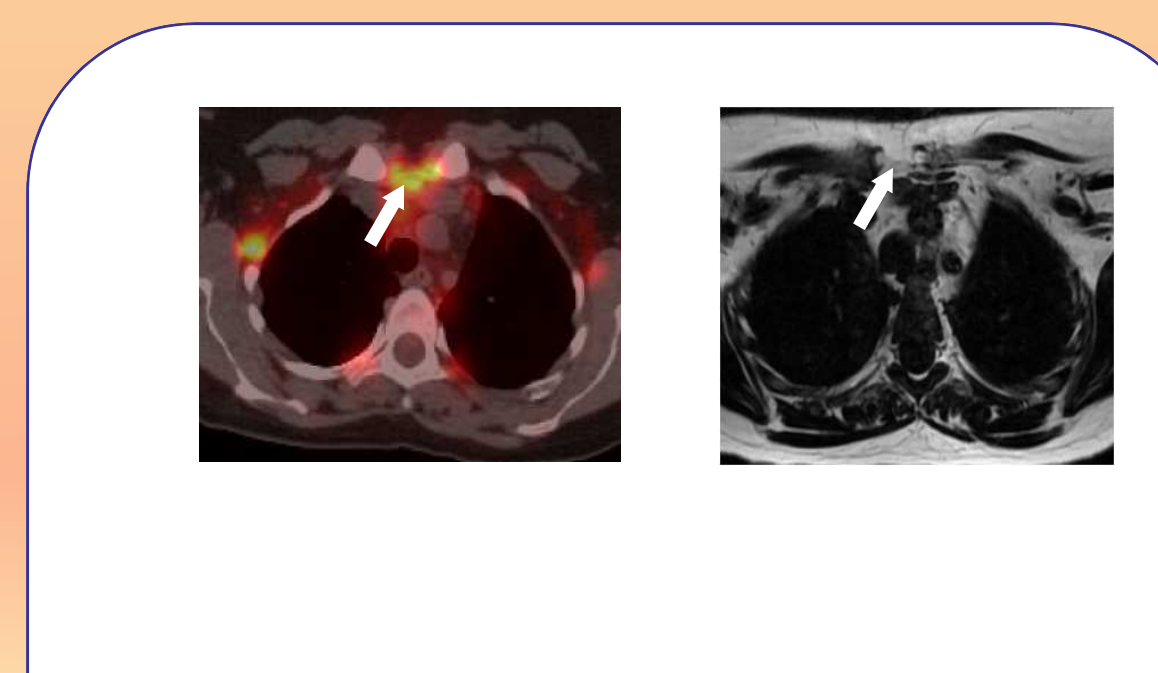

Figure 2: (a) ${ }^{18}$ F-FDG uptake within the suprasternal notch on PET-CT (arrow); (b) fat:IDEAL MR at the same level showing corresponding low signal in the

suprasternal notch; (c) Haematoxylin-Eosin staining (d) UCP1 immunostaining

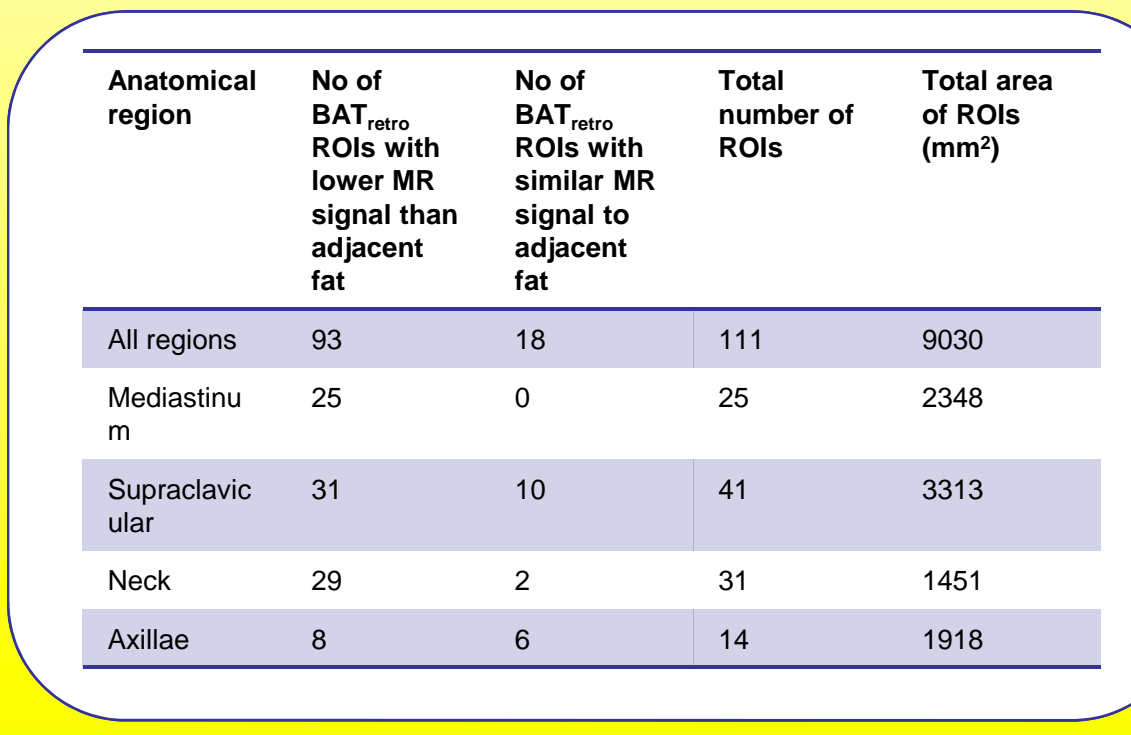

\section{Conclusion}

We provide the first ever report that MR can be used reliably to identify BAT in a human adult, with histological and immunohistochemical confirmation. Our data demonstrate proof of concept to support the development of MR, a safe and reproducible imaging modality, as a biomarker for human BAT.

Funded by: Warwick Strategic Grant- University of Warwick Acknowledgements:

Sean James, Dept of Histopathology, UHCW. UHCW Radiographers 